\title{
Quasilinear analysis of loss-cone driven weakly relativistic electron cyclotron maser instability
}

Cite as: Physics of Plasmas 2, 1285 (1995); https://doi.org/10.1063/1.871459

Submitted: 03 October 1994 . Accepted: 03 January 1995. Published Online: O3 September 1998

L. F. Ziebell, and Peter H. Yoon

\section{ARTICLES YOU MAY BE INTERESTED IN}

Loss-cone instability: Wave saturation by particle trapping

Physics of Plasmas 14, 122302 (2007); https://doi.org/10.1063/1.2799621

Gyroharmonic maser instability for weakly relativistic electrons with a loss-cone distribution

Physics of Fluids B: Plasma Physics 2, 1918 (1990); https://doi.org/10.1063/1.859463

Quasi-linear theory of cyclotron maser instability

The Physics of Fluids 22, 1811 (1979); https://doi.org/10.1063/1.862786 


\title{
Quasilinear analysis of loss-cone driven weakly relativistic electron cyclotron maser instability
}

\author{
L. F. Ziebell \\ Instituto de Física, UFRGS, Caixa Postal 15051, 91501-970 Porto Alegre, RS, Brazil \\ Peter H. Yoon \\ Institute for Physical Science and Technology, University of Maryland, College Park, Maryland 20742
}

(Received 3 October 1994; accepted 3 January 1995)

\begin{abstract}
This paper presents a quasilinear analysis of the relativistic electron cyclotron maser instability. Two electron populations are assumed: a low-temperature background component and a more energetic loss-cone population. The dispersion relation is valid for any ratio of the energetic to cold populations, and includes thermal and relativistic effects. The quasilinear analysis is based upon an efficient kinetic moment method, in which various moment equations are derived from the particle kinetic equation. A model time-dependent loss-cone electron distribution function is assumed, which allows one to evaluate the instantaneous linear growth rate as well as the moment kinetic equations. These moment equations along with the wave kinetic equation form a fully self-consistent set of equations which governs the evolution of the particles as well as unstable waves. This set of equations is solved with physical parameters typical of the earth's auroral zone plasma. (c) 1995 American Institute of Physics.
\end{abstract}

\section{INTRODUCTION}

The relativistic electron cyclotron maser instability is driven by an inverse population of electrons in momentum space perpendicular to the magnetic field. The instability operates near the electron cyclotron frequency and/or its harmonics, and unlike most plasma instabilities the maser instability amplifies various radiation modes as well as the usual trapped modes. The instability relies on the relativistic wave-particle interaction. Without the relativistic effect, the maser instability cannot be described. Because of these characteristics, the instability is called the relativistic electron cyclotron maser in the literature.

During the past decade or so, extensive studies of the relativistic electron cyclotron maser instability have been carried out. ${ }^{1-38}$ These authors have applied the relativistic maser instability to a wide variety of applications ranging from astrophysical radio sources, ${ }^{1-3}$ planetary radio emissions, ${ }^{4-8}$ solar microwave bursts, ${ }^{9-13}$ the earth's kilometric radiation, ${ }^{14-36}$ and laboratory microwave generation devices. ${ }^{37,38}$

Among these possible applications, we mention a wellknown example, namely, the earth's kilometric radiation. This radiation phenomenon was successfully explained in terms of the weakly relativistic maser instability by $\mathrm{Wu}$ and Lee. ${ }^{14}$ Their work stimulated the recent surge of research activities on this topic in the space and astrophysical plasma physics community. The source of instability for the kilometric radiation was identified as the loss-cone electrons that are formed when the energetic electrons injected from the geomagnetic tail region are reflected by the converging geomagnetic field.

Many of the articles in the literature on this instability emphasize the linear aspect ${ }^{14-28}$ (for an extensive review of the topic see Ref. 29) although a few discussions on nonlinear evolution can also be found in the literature. Most nonlinear theories make use of numerical simulation method. ${ }^{30-33}$ Some attempts have been made, however, to study the nonlinear stage of the instability by using analytical means, namely quasilinear theory. ${ }^{11,12,15,28,36}$ Studies based upon particle simulations have confirmed that the dominant saturation mechanism for the maser instability is the quasilinear process.

Among these works related to quasilinear theory, Refs. 11 and 36 discuss the instantaneous anomalous transport rate (i.e., quasilinear diffusion rate) that results from the maser instability. Particularly, Ref. 11 discusses the case of dominant isotropic background plasma with a small component of loss-cone electrons, while Ref. 36 discusses the case when the background electron component is absent. On the other hand, Ref. 15 and its generalization Ref. 28 discuss the solution of the quasilinear equation by assuming a quasi or exactly perpendicular propagation angle. A recent work by Aschwanden, ${ }^{12}$ however, constitutes an important step toward a comprehensive treatment of quasilinear theory of maser instability without employing various approximations which were utilized in other related works. ${ }^{11,15,28,36}$ Aschwanden numerically solved the set of fully selfconsistent quasilinear particle and wave kinetic equations, on the basis of cold plasma dispersion relation and the assumption that cold electrons support the wave while the energetic population contributes to the growth of the wave.

The present paper resumes the subject by using a dispersion relation in which thermal effects as well as effects of energetic population on the dispersive property of the wave are fully taken into account. Whereas Aschwanden solved the particle kinetic equation numerically, we make use of an efficient moment kinetic equation by modeling an appropriate time-dependent particle distribution function. This method has already been employed successfully in previous studies, ${ }^{39,40}$ with the use of cold plasma dispersion relation, and now assumes a full power with the inclusion of thermal effects. Therefore, this paper introduces a new and efficient 
method for quasilinear analysis of the maser instability. As an application, we choose one set of parameters typical of the earth's auroral zone plasma. Of course, more elaborate discussion with an extensive parameter study can be carried out on the basis of the present method.

The organization of this paper is the following. Section II presents the general formulation employed in the present investigation, including the dispersion relation for electromagnetic waves, the procedure for obtaining the linear growth rate, and quasilinear formalism utilized in the present work. Thermal and relativistic effects are fully taken into account in the derivation of the dielectric tensor elements. Then, Sec. III introduces the model time-dependent electron loss-cone distribution function, based on which the moment kinetic equations are explicitly evaluated. The general formalism developed in Sec. II is then applied to this particular model. Section IV is dedicated to a numerical analysis, and finally, Sec. V presents summary and discussion.

\section{GENERAL FORMULATION}

In the present analysis we are interested in the instabilities that operate in the vicinity of the electron cyclotron frequency and/or its harmonics. Consequently, because of the high wave frequency, the ions are treated as if they are infinitely massive so that the ion response to any perturbation can be ignored. Hence, the ions play no dynamical role but only provide an overalt charge neutrality.

The electrons are assumed to be composed of relatively less energetic (i.e., "cold") component and an energetic component possessing a one-sided loss-cone structure in momentum space. The ambient magnetic field is assumed to lie in $z$ direction $\left(\mathbf{B}_{0}=B_{0} \mathbf{e}_{z}\right)$, and the wave vector $\mathbf{k}$ lies in $x-z$ plane, $\mathbf{k}=k \sin \theta \mathrm{e}_{x}+k \cos \theta \mathrm{e}_{z}$.

\section{A. Dispersion relation}

The dispersion relation is written as

$$
\Lambda(\mathbf{k}, \omega)=A N_{\perp}^{4}+B N_{\perp}^{2}+C=0,
$$

where

$$
\begin{aligned}
A= & \left(1+\chi_{11}\right)\left(1-\chi_{33}\right)+\chi_{13}\left(2 N_{\|}+\chi_{13}\right)+\chi_{33} N_{\|}^{2}, \\
B= & \left(\chi_{11}+1-N_{\|}^{2}\right)\left[\chi_{23}^{2}-1-e_{33}+\chi_{33}\left(\chi_{22}+1-N_{\|}^{2}\right)\right] \\
& -\left(\chi_{22}+1-N_{\|}^{2}\right)\left[1+\chi_{11}+\chi_{13}\left(2 N_{\|}+\chi_{13}\right)\right] \\
& -\chi_{12}^{2}\left(1-\chi_{13}\right)+2 \chi_{12} \chi_{23}\left(N_{\|}+\chi_{13}\right), \\
C= & \left(1+e_{33}\right)\left[\left(\chi_{11}+1-N_{\| 1}^{2}\right)\left(\chi_{22}+1-N_{\|}^{2}\right)+\chi_{12}^{2}\right] .
\end{aligned}
$$

$N_{\perp}$ and $N_{\|}$are, respectively, the perpendicular and parallel components of $\mathbf{N}=c \mathbf{k} / \omega$, where $\mathbf{k}$ is the wave vector. We use indices $(1,2,3)$ and $(x, y, z)$ interchangeably.
The tensor $\chi_{i j}$ whose elements appear in Eq. (1) are defined as part of the dielectric tensor, which may be written with the use of a double series expansion on both harmonics of the cyclotron frequency and Larmor radius, as in Ref. 41. These infinite series can be rewritten as a single infinite series, ${ }^{35}$ resulting in the following expression:

$$
\epsilon_{i j}=\delta_{i j}+\delta_{i 3} \delta_{j 3} e_{33}+N_{\perp}^{\delta_{i 3}+\delta_{j 3}} \chi_{i j},
$$

where

$$
\begin{aligned}
e_{33}= & X \int d^{3} u \frac{u_{\|} \partial f / \partial u_{\|}}{\gamma-N_{\|} u_{\|}}, \\
\chi_{i j}= & \frac{X}{Y^{\delta_{i 3}+\delta_{j 3}}} \sum_{m=1}^{\infty}\left(\frac{N_{\perp}}{Y}\right)^{2(m-1)} \\
& \times \sum_{n=-m}^{m} s_{i j}(n, m) I\left(n, m, \delta_{i 3}+\delta_{j 3}\right) .
\end{aligned}
$$

In these expressions, we have defined

$$
\begin{aligned}
& X=\frac{\omega_{\mathrm{pe}}^{2}}{\omega^{2}}, \quad Y=\frac{\left|\Omega_{e}\right|}{\omega}, \\
& I(n, m, l)=\int d^{3} u \frac{u_{||}^{l} u_{\perp}^{2 m-1}}{\gamma-n Y-N_{\|} u_{\|}} \mathscr{J} f \\
& s_{11}(n, m)=n^{2} a(|n|, m-|n|), \\
& s_{12}(n, m)=-i m n a(|n|, m-|n|)=-s_{21}(n, m), \\
& s_{13}(n, m)=n a(|n|, m-|n|)=s_{31}(n, m), \\
& s_{22}(n, m)=b(|n|, m-|n|), \\
& s_{23}(n, m)=i m a(|n|, m-|n|)=-s_{32}(n, m), \\
& s_{33}(n, m)=a(|n|, m-|n|), \\
& a(n, m)=\frac{(-1)^{m}[2(n+m)] !}{[(n+m) !]^{2}(2 n+m) ! m !}\left(\frac{1}{2}\right)^{2(n+m)}, \\
& b(0, m)=a(1, m-2), \\
& b(n, m)=\frac{1}{4}[a(n-1, m)+a(n+1, m-2) \\
& \left.\quad-2 \frac{n+m-1}{n+m} a(n, m-1)\right], \quad n>0,
\end{aligned}
$$

with an additional rule $[(1) /(-m) !]=0$, for $m \geqslant 1$.

Moreover,

$$
\mathscr{L}=\frac{1}{\gamma}\left[\left(\gamma-N_{\|} u_{\|}\right) \partial_{u_{\perp}}+N_{\|} u_{\perp} \partial_{u_{\|}}\right]
$$

where $\mathbf{u}=\mathbf{p} /(m c)$. Finally, $\omega_{\mathrm{pe}}$ is the electron plasma frequency and $\Omega_{e}$ is the electron cyclotron frequency.

\section{B. Quasilinear formalism}

Quasilinear kinetic equation for the energetic electron component may be derived in a standard textbook procedure, and may be written as follows: 


$$
\partial_{t} f_{e}=\frac{1}{u_{\perp}} L^{\prime}\left(u_{\perp} \frac{\pi e^{2}}{m_{e}^{2} c^{2} \gamma} \sum_{n=-\infty}^{\infty} \int \frac{d^{3} k}{|\omega|}\left|\delta E_{\mathbf{k}}\right|^{2}\left|\hat{\mathbf{a}}_{\mathbf{k}} \cdot \mathbf{\Pi}\right|^{2} \delta\left(\gamma-n Y-N_{\|} u_{\|}\right)\right) L f_{e}
$$

where summation over unstable modes is implicit, and

$$
\begin{aligned}
& L^{\prime}=n Y \partial_{u_{\perp}}+\partial_{u_{\|}} N_{\|} N_{\perp}, \\
& \begin{aligned}
L=n Y \partial_{u_{\perp}} & +N_{\|} u_{\perp} \partial_{u_{\|}}, \\
& \hat{\mathrm{a}}_{\mathrm{k}} \cdot \Pi=(-1)^{n}\left\{\left[\epsilon_{12}\left(\epsilon_{33}-N_{\perp}^{2}\right)+\epsilon_{23}\left(\epsilon_{13}+N_{\|} N_{\perp}\right)\right] \Pi_{1}^{n}+\left[\left(\epsilon_{13}+N_{\|} N_{\perp}\right)^{2}-\left(\epsilon_{11}-N_{\|}^{2}\right)\left(\epsilon_{33}-N_{\perp}^{2}\right)\right] \Pi_{2}^{n}-\left[\epsilon_{23}\left(\epsilon_{11}-N_{\|}^{2}\right)\right.\right. \\
& \left.\left.+\epsilon_{12}\left(\epsilon_{13}+N_{\perp} N_{\|}\right)\right] \Pi_{3}^{n}\right\} \times\left\{-\left[\epsilon_{12}\left(\epsilon_{33}-N_{\perp}^{2}\right)+\epsilon_{23}\left(\epsilon_{13}+N_{\perp} N_{\|}\right)\right]^{2}+\left[\left(\epsilon_{13}+N_{\|} N_{\perp}\right)^{2}-\left(\epsilon_{11}-N_{\|}^{2}\right)\left(\epsilon_{33}-N_{\perp}^{2}\right)\right]^{2}\right. \\
& \left.\quad-\left[\epsilon_{23}\left(\epsilon_{11}-N_{\|}^{2}\right)+\epsilon_{12}\left(\epsilon_{13}+N_{\perp} N_{\|}\right)\right]^{2}\right\}^{-1 / 2},
\end{aligned} \\
& \quad \Pi_{1}^{n}=-\frac{n}{\lambda} J_{n}(\lambda), \quad \Pi_{2}^{n}=i J_{n}^{\prime}(\lambda), \quad \Pi_{3}^{n}=\frac{u_{\|}}{u_{\perp}} J_{n}(\lambda), \quad \lambda=\frac{-N_{\perp} u_{\perp}}{Y} .
\end{aligned}
$$

Defining $u$ and $\mu$ variables, such that $u \equiv\left(u_{\perp}^{2}+u_{\|}^{2}\right)^{1 / 2}$ and $\mu \equiv u_{\|}\left(u_{\perp}^{2}+u_{\|}^{2}\right)^{-1 / 2}$, and defining moments of quasilinear equation as $\langle g\rangle \equiv \int d^{3} u g f_{e}$, we arrive at the following:

$$
\begin{aligned}
& \frac{d}{d t}\left\langle u^{2}\right\rangle=-2 \int d^{3} u\left(1-\mu^{2}\right) u\left(D_{u u} \partial_{u} f_{e}-\frac{D_{u \mu}}{u} \partial_{\mu} f_{e}\right), \\
& \frac{d}{d t}\langle\mu\rangle=\int d^{3} u \frac{1-\mu^{2}}{u}\left(D_{\mu u} \partial_{u} f_{e}-\frac{D_{\mu \mu}}{u} \partial_{\mu} f_{e}\right),
\end{aligned}
$$

where

$$
D_{a b}=\frac{\pi e^{2}}{m_{e}^{2} c^{2}} \sum_{n=-\infty}^{\infty} \int d^{3} k\left|\delta E_{\mathrm{k}}\right|^{2}\left|\hat{\mathbf{a}}_{\mathrm{k}} \cdot \Pi\right|^{2} \frac{\gamma}{|\omega|} \delta\left(\gamma-n Y-N_{\|} u_{\|}\right) \Delta_{a} \Delta_{b}, \quad \Delta_{u}=1, \quad \Delta_{\mu}=\mu-\frac{N_{\|} u}{\gamma} .
$$

These expressions for the diffusion coefficient tensor $D_{a b}$ can be substituted into Eq. (4) explicitly. As a consequence, one can show that the time evolution of the moments are given by

$$
\begin{aligned}
\frac{d}{d \tau}\left\langle u^{2}\right\rangle= & -4 \pi^{2} \sum_{n=-\infty}^{\infty} \int_{-\infty}^{\infty} d q \int_{0}^{\infty} d w\left|\frac{\delta E_{w q}}{B_{0}}\right|^{2} Y \int d^{3} u\left(1-\mu^{2}\right) u\left|\hat{\mathbf{a}}_{\mathrm{k}} \cdot \mathbf{\Pi}\right|^{2}\left[\partial_{u} f_{e}-\frac{1}{u}\left(\mu-\frac{N_{\|} u}{\gamma}\right) \partial_{\mu} f_{e}\right] \gamma \delta\left(\gamma-n Y-N_{\|} u_{\|}\right) \\
\frac{d}{d \tau}\langle\mu\rangle= & 2 \pi^{2} \sum_{n=-\infty}^{\infty} \int_{-\infty}^{\infty} d q \int_{0}^{\infty} d w\left|\frac{\delta E_{w q}}{B_{0}}\right|^{2} Y \int d^{3} u \frac{\left(1-\mu^{2}\right)}{u}\left(\mu-\frac{N_{\|} u}{\gamma}\right)\left|\hat{\mathbf{a}}_{\mathrm{k}} \cdot \mathbf{\Pi}\right|^{2}\left[\partial_{u} f_{e}-\frac{1}{u}\left(\mu-\frac{N_{\|} u}{\gamma}\right) \partial_{\mu} f_{e}\right] \\
& \times \gamma \delta\left(\gamma-n Y-N_{\|} u_{\|}\right),
\end{aligned}
$$

where we have defined the normalized time $\tau \equiv\left|\Omega_{e}\right| t$, and have rewritten the spectral wave energy density as a function of normalized quantities

$$
\begin{aligned}
& \int d^{3} k\left|\delta E_{\mathrm{k}}\right|^{2}=2 \pi \int_{-\infty}^{\infty} d q \int_{0}^{\infty} d w\left|\delta E_{w q}\right|^{2}, \\
& q=\frac{c k_{\|}}{\left|\Omega_{e}\right|}, \quad w \equiv \frac{\omega}{\left|\Omega_{e}\right|} .
\end{aligned}
$$

The quantity $N_{\perp}$ that appears in $\left|\hat{\mathbf{a}}_{\mathbf{k}} \cdot \mathbf{I I}\right|^{2}$ is obtained from the dispersion relation, as a function of $N_{\|}$and $w$, where $N_{\|}=q / w$.

Using the resonance condition, $u$ integration can be performed, and the time evolution of the moments can be shown to reduce to

$$
\begin{aligned}
\frac{d}{d \tau}\left\langle u^{2}\right\rangle= & -8 \pi^{3} \sum_{n=-\infty}^{\infty} \int_{-\infty}^{\infty} d q \int_{0}^{\infty} d w\left|\frac{\delta E_{w q}}{B_{0}}\right|^{2} Y\left(\Theta(n Y-1) \int_{-1}^{1} d \mu Q_{+}(\mu)+\Theta(1-n Y) \Theta\left(n^{2} Y^{2}-1+N_{\|}^{2}\right)\right. \\
& \left.\times \int_{\mu_{i}}^{\mu_{f}} d \mu\left[Q_{+}(\mu)+Q_{-}(\mu)\right]\right)
\end{aligned}
$$




$$
\begin{aligned}
\frac{d}{d \tau}\langle\mu\rangle= & 4 \pi^{3} \sum_{n=-\infty}^{\infty} \int_{-\infty}^{\infty} d q \int_{0}^{\infty} d w\left|\frac{\delta E_{w q}}{B_{0}}\right|^{2} Y\left[\Theta(n Y-1) \int_{-1}^{1} d \mu Q_{+}(\mu) \frac{\mu-N_{\|} u_{+} / \gamma_{+}}{u_{+}^{2}}+\Theta(1-n Y) \Theta\left(n^{2} Y^{2}-1\right.\right. \\
& \left.\left.+N_{\|}^{2}\right) \int_{\mu_{i}}^{\mu_{f}} d \mu\left(Q_{+}(\mu) \frac{\mu-N_{\|} u_{+} / \gamma_{+}}{u_{+}^{2}}+Q_{-}(\mu) \frac{\mu-N_{\|} u_{-} / \gamma_{-}}{u_{-}^{2}}\right)\right],
\end{aligned}
$$

where

$$
\begin{gathered}
Q_{s}(\mu)=\frac{\left(1-\mu^{2}\right) u_{s}^{2} \gamma_{s}\left|\hat{\mathbf{a}}_{\mathbf{k}} \cdot \mathbf{\Pi}\right|^{2}}{\left|u_{s}-N_{\|} \mu \gamma_{s}\right|}\left[\gamma u \partial_{u} f_{e}+\left(N_{\|} u-\gamma \mu\right) \partial_{\mu} f_{e}\right]_{u_{s}}, \quad u_{s}=\frac{n Y N_{\|} \mu+s \sqrt{n^{2} Y^{2} N_{\|}^{2} \mu^{2}-\left(1-n^{2} Y^{2}\right)\left(1-N_{\|}^{2} \mu^{2}\right)}}{1-N_{\|}^{2} \mu^{2}}, \\
s= \pm 1 .
\end{gathered}
$$

The upper and lower limits in the $\mu$ integration are given by $\mu_{i}=\sqrt{1-n^{2} Y^{2}} / N_{\|}, \quad \mu_{f}=1$, for $N_{\|}>0$, and $\mu_{i}=-1$, $\mu_{f}=-\sqrt{1-n^{2} Y^{2}} /\left|N_{\|}\right|$, for $N_{\|}<0$.

The wave amplitudes evolve in time according to

$$
\frac{d}{d \tau}\left|\delta E_{w q}\right|^{2}=2 w_{i}\left|\delta E_{w q}\right|^{2},
$$

where the normalized growth rate $w_{i}=\operatorname{Im}(w)$ is selfconsistently obtained from the dispersion relation (1).

\section{Derivation of the growth rates}

The instantaneous growth rate, which is necessary for quasilinear evolution of the wave is obtained from the solution of the dispersion relation. However, the dielectric tensor which appears in the coefficients of Eq. (1) when thermal effects are taken into account, features a rather complex dependence on $\omega$. As a consequence, to obtain the wave frequency as a function of $\mathbf{k}$, for a given mode, is not a trivial task, and requires sophisticated and time-consuming numerical procedures. In order to overcome this difficulty and to make the present quasilinear treatment tractable, we employ an approximate procedure, which turns out to be quite satisfactory as far as the frequency range in which significant growth occurs is not in the range of anomalous dispersion, and as far as the group velocity does not approach zero.

We obtain the growth rate as follows. The dispersion relation as given by Eq. (1) is formally written as a quartic equation, with $N_{\perp}$ as the unknown. Therefore, the roots can be formally written as

$$
N_{\perp}^{2}=\frac{-B \pm \sqrt{B^{2}+4 A C}}{2 A} .
$$

This is a formal expression, since the coefficients $A, B$, and $C$ are functions of $N_{\perp}$. We then follow an iterative procedure. For a given value of the real wave frequency, we evaluate the cold plasma root corresponding to a specific mode. This root is inserted into the coefficients $A, B$, and $C$, and new roots are found from Eq. (8). In this procedure, the quantity $N_{\|}$is treated as a parameter. It can be proved $a$ posteriori that the most important wave growth occurs in directions almost perpendicular to the ambient magnetic field. With sufficient accuracy, therefore, $N_{\|}$can be considered as real along the ray path. The new roots found are then substituted to the coefficients, and the process is repeated until convergence is achieved. We found that usually only a few iterations are sufficient to attain convergence.

The outcome of this iterative procedure is a complex value of $N_{\perp}$ (or $k_{\perp}$, since these quantities are proportional). For a finite group velocity, negative imaginary part of the wave vector implies that the wave amplitude grows convectively. Owing to the finite group velocity, the growth along the ray path can be written equivalently as a temporal growth. This leads to the following approximate expression for the growth rate:

$$
\omega_{i} \simeq-k_{i} v_{g x} .
$$

In the above, $v_{g x}$ is the $x$ component of the group velocity, and $k_{i}$ is the imaginary part of $k_{\perp}$.

\section{MODEL TIME-DEPENDENT ELECTRON LOSS- CONE DISTRIBUTION}

The electron population in the source region is assumed to be composed of two populations. The cold background population is represented by a low temperature Maxwellian distribution function

$$
f_{b}(u, \mu)=\frac{1}{\pi^{3 / 2} \alpha_{b}^{3}(t)} \exp \left(-\frac{u^{2}}{\alpha_{b}^{2}(t)}\right)
$$

where $\alpha_{b}^{2}=2 T_{b} / m_{e} c^{2}$, and $T_{b}$ is the temperature of the background electrons, measured in energy units. The background distribution is assumed to be stationary during the time evolution of the system.

We now proceed to discuss the model distribution function for the energetic electrons possessing a one-sided loss cone. The model distribution is assumed to be valid for all times. That is, instead of solving the kinetic equation for the distribution function, we simplify the analysis considerably by assuming a time-dependent model for the loss-cone electron component. For the present purpose, we consider the following model distribution function: ${ }^{39.40}$

$$
f_{e}(u, \mu, t)=\frac{1}{\pi^{3 / 2} \alpha^{3}(t)} \exp \left(-\frac{u^{2}}{\alpha^{2}(t)}\right) G(\mu, t) .
$$

The total thermal energy associated with the energetic loss-cone electrons is given by $E / m c^{2}=n_{e}\left\langle u^{2} / 2\right\rangle$ $=3 n_{e} \alpha^{2} / 4$, where $\langle\cdots\rangle=2 \pi \int_{-1}^{1} d \mu \int_{0}^{\infty} d u u^{2} \cdots f(u, \mu)$. 
Using the relation $E=3 n_{e} T_{e} / 2$, one can relate the effective temperature of the loss-cone electrons to the parameter $\alpha$ as $T_{e}=m_{e} c^{2} \alpha^{2} / 2$.

In Eq. (11) $G(\mu, t)$ is the time-dependent pitch angle distribution modeled by

$G(\mu, t)=\frac{1}{1+\Delta(t)-\delta \tanh (1 / \delta)}\left[\tanh ^{2}\left(\frac{\mu-1}{2 \delta}\right)+\Delta(t)\right]$.

The loss-cone angle $\theta_{\mathrm{LC}}$ can be determined by taking the second derivative of the function $G(\mu, t)$ with respect to $\mu$ and setting it equal to zero. It turns out that the loss-cone angle $\theta_{\mathrm{LC}}$ is given by

$$
\theta_{\mathrm{LC}}=\cos ^{-1}[1+\delta \ln (2-\sqrt{3})] \text {. }
$$

For $\theta_{\mathrm{LC}}=15^{\circ}$ the corresponding $\delta=0.025$, for $\theta_{\mathrm{LC}}=30^{\circ}$, $\delta=0.1017$, for $\theta_{\mathrm{LC}}=45^{\circ}, \delta=0.2224$, for $\theta_{\mathrm{LC}}=60^{\circ}, \delta=0.3797$, for $\theta_{\mathrm{LC}}=75^{\circ}, \delta=0.5628$, and for $\theta_{\mathrm{LC}}=90^{\circ}, \delta=0.7593$.

Shown in Fig. 1 are three-dimensional surface plots as well as two-dimensional contour plots of the model distribution function for energetic electrons, for $T_{e}=4 \mathrm{keV}$, losscone angle of $\theta_{\mathrm{LC}}=30^{\circ}$ (corresponding to $\delta=0.1017304$ ) and for three different values of the parameter $\Delta(\Delta=0,1$, and 10). It should be noted that the limit $\Delta \rightarrow \infty$ corresponds to an isotropic distribution with no loss-cone feature.

The averages $\left\langle u^{2}\right\rangle$ and $\langle\mu\rangle$, whose evolution in time is governed by Eqs. (6), are related to the parameters $\alpha$ and $\Delta$ as follows, which can be easily demonstrated:

$$
\frac{d \alpha^{2}}{d t}=\frac{2}{3} \frac{d\left\langle\mu^{2}\right\rangle}{d t}, \quad \frac{d \Delta}{d t} \approx \frac{(1+\Delta)^{2}}{\delta} \frac{d\langle\mu\rangle}{d t} .
$$

These relations are used in the numerical study of quasilinear evolution. Another useful notion, which is introduced as this point, is the notion of the ratio between wave energy and particle energy (associated with the energetic electrons), for each wave mode

$$
\begin{aligned}
\frac{E_{\text {wave }}}{E_{\text {particle }}}= & \frac{\int d^{3} k\left(\left|\delta E_{\mathrm{k}}\right|^{2}+\left|\delta B_{\mathrm{k}}\right|^{2}\right) /(8 \pi)}{n_{e} m_{e} c^{2} \int d^{3} u\left(u^{2} / 2\right) f_{e}}, \\
= & \frac{4 \pi}{3 \alpha^{2}}\left(1+\frac{n_{b}}{n_{e}}\right) \frac{\Omega_{e}^{2}}{\omega_{p}^{2}} \int_{-\infty}^{\infty} d q \int_{0}^{\infty} d w\left|\delta E_{w q}\right|^{2} \\
& \times\left[1+|\mathbf{N}|^{2}\left(1-\left|\hat{\mathbf{a}}_{\mathrm{k}} \cdot \hat{\mathbf{k}}\right|^{2}\right)\right],
\end{aligned}
$$

where $\hat{k}$ is a unit wave vector. This quantity is useful for the normalization of the wave spectra.

\section{A. Evaluation of $I(n, m, I)$ and $e_{33}$ for the energetic distribution}

The $I(n, m, l)$ integrals defined in' Eq. (2) can be separated into principal and resonant parts, by the use of the Landau contour

$$
\begin{aligned}
& I(n, m, l)=I^{\prime}(n, m, l)+i I^{\prime \prime}(n, m, l), \\
& I^{\prime}(n, m, l)=\mathscr{P} \int d^{3} u \frac{u_{\|}^{l} u_{\perp}^{2 m-1}}{\gamma-n Y-N_{\|} u_{\|}} \mathscr{E} f_{e}, \\
& I^{\prime \prime}(n, m, l)=-\pi \int d^{3} u u_{\|}^{l} u_{\perp}^{2 m-1} \mathscr{E} f_{e} \delta\left(\gamma-n Y-N_{\|} u_{\|}\right),
\end{aligned}
$$
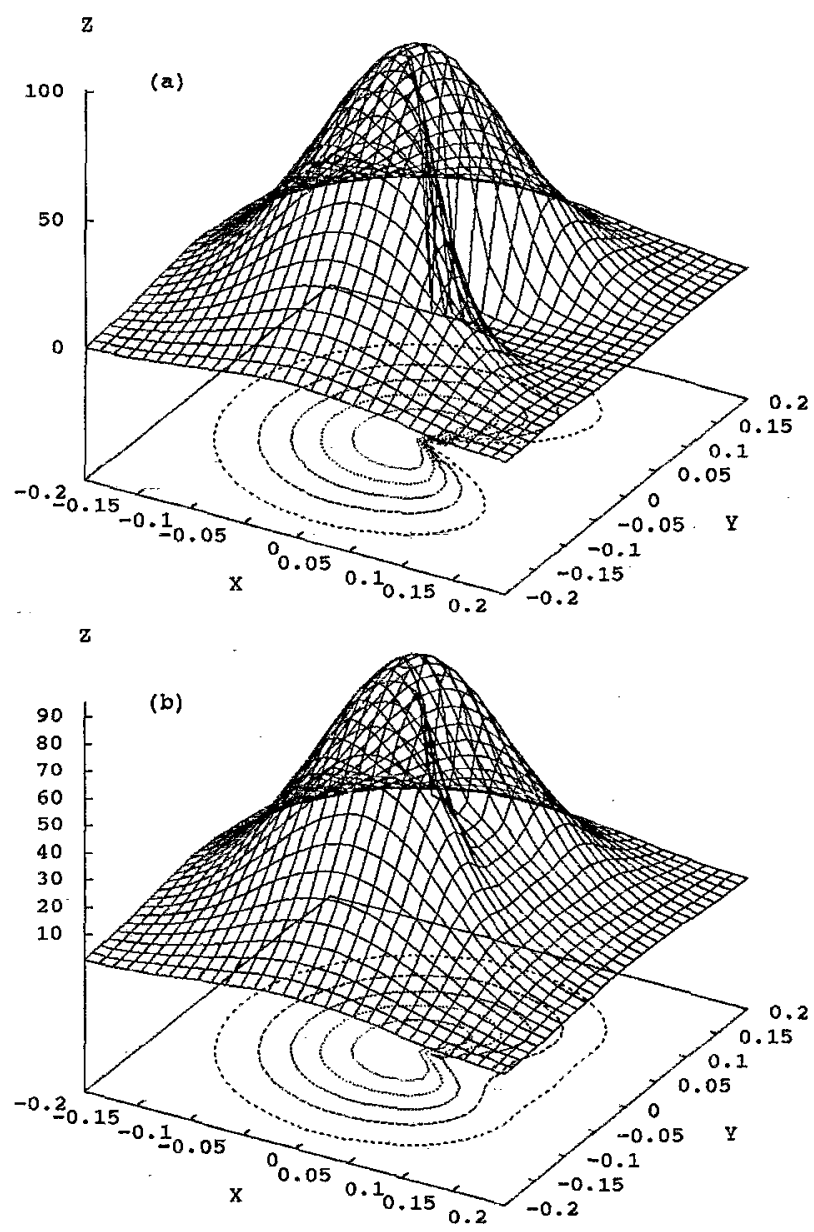

$\mathrm{z}$

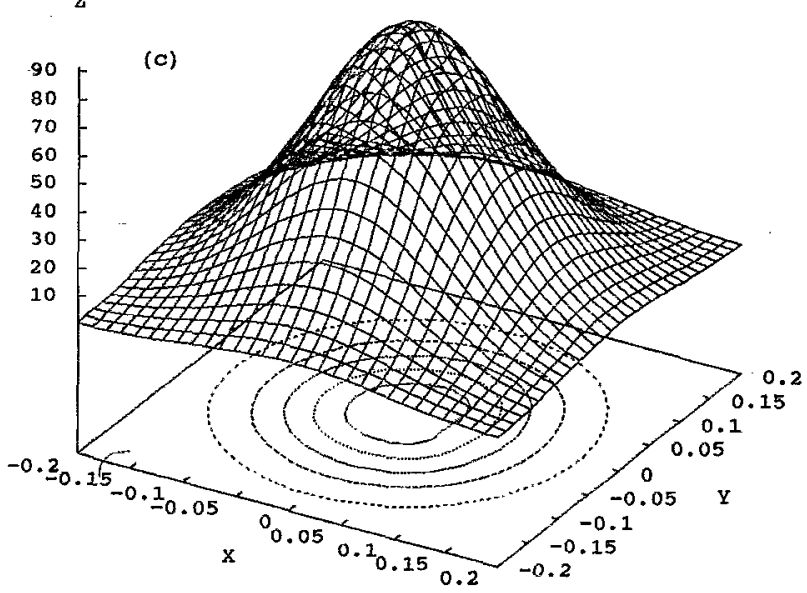

FIG. 1. Three-dimensional surface plots as well as two-dimensional contour plots of the model distribution function for energetic electrons, for $\theta_{\mathrm{LC}}=30^{\circ}$ and $T_{e}=4 \mathrm{keV}$. (a) $\Delta=0$, (b) $\Delta=1$, and (c) $\Delta=10$.

where 9 stands for the principal part of the integral.

Let us first discuss the case of energetic electrons, whose distribution is described by Eq. (11). The resonant part $I^{\prime \prime}(n, m, l)$ may be written as follows:

$$
\begin{aligned}
I^{\prime \prime}(n, m, l)= & -2 \pi^{2}\left(\Theta(n Y-1) \int_{-1}^{1} d \mu P_{+}(\mu)\right. \\
& +\Theta(1-n Y) \Theta\left(n^{2} Y^{2}-1+N_{\|}^{2}\right)
\end{aligned}
$$




$$
\left.\times \int_{\mu_{i}}^{\mu_{f}} d \mu\left[P_{+}(\mu)+P_{-}(\mu)\right]\right)
$$

where

$$
\begin{aligned}
P_{s}(\mu)= & \frac{\mu^{l}\left(1-\mu^{2}\right)^{m} u_{s}^{h+2 m+1}}{\left|u_{+}-N_{\|} \mu \gamma_{+}\right|}\left[\gamma \partial_{u} f_{e}+\left(N_{\|}\right.\right. \\
& \left.-\gamma \mu / u) \partial_{\mu} f_{e}\right]_{u_{s}}
\end{aligned}
$$

The symbol $[\cdots]_{u_{s}}$ means that the quantity has to be evaluated at the resonant velocity $u_{s}$. The derivatives $\partial_{u} f_{e}$ and $\partial_{\mu} f_{e}$ can be obtained in a straightforward manner from Eq. (11). The limits of the integration, $\mu_{i}$ and $\mu_{f}$, are the same as defined immediately following Eq. (6).

The growth rate is very sensitive to changes in the distribution function along the resonance curve in momentum space. Therefore, the resonant parts, as given by $\mathrm{Eq}$. (17), must be evaluated self-consistently at each time step along the time evolution of the distribution. On the other hand, the principal parts are dependent upon the integrated distribution and usually can be regarded as rather insensitive to detailed features in the distribution. unless some peculiar circumstances are satisfied. ${ }^{42}$ This virtual independence on detailed features of the distribution allows for some further approximations, which simplify the evaluation of the principal parts of the integral, and contribute very much to speeding up the quasilinear code. With this reasoning taken into account, when evaluating the principal part of the integral $I(n, m, l)$ we neglect the $\mu$ dependence of the distribution, thereby considering a Maxwellian distribution. Moreover, to avoid repeated evaluations, we consider that the temperature of the distribution do not change appreciably along the time evolution. Therefore, the principal part of the integral can be regarded as a constant. The validity of this approximation is confirmed a posteriori with an actual observation of the temperature change in the numerical analysis that follows.

Therefore, the principal part of the integral $I(n, m, l)$, for the case of energetic population is given by

$$
\begin{aligned}
I^{\prime}(n, m, l)= & -\frac{4 \alpha_{0}^{2 m-2}}{\sqrt{\pi}} \int_{-\infty}^{\infty} d u_{\|} u_{\|}^{\prime} e^{-\mu_{e} u_{\|}^{2} / 2} \\
& \times\left(\Theta(m) \sum_{k=0}^{m-1}(m-1-k) ! x_{n}^{k}\right. \\
& \left.-x_{n}^{m} e^{-x_{n}} \operatorname{Ei}\left(x_{n}\right)\right),
\end{aligned}
$$

where $x_{n}=-\left[(2) /\left(\alpha_{0}^{2}\right)\right]\left(1-n Y-N_{\|} u_{\|}+u_{\|}^{2} / 2\right)$. Here, $E i(x)$ is the exponential integral, ${ }^{43}$ defined by

$$
E i(x)=-\not \int_{-x}^{\infty} d t \frac{e^{-t}}{t} .
$$

The quantity $e_{33}$ can be written in a similar way

$$
e_{33}=\frac{4}{\alpha_{0}^{2}} \frac{X_{e}}{\sqrt{\pi}} \int_{-\infty}^{\infty} d u_{\|} u_{\|}^{2} e^{-\mu_{e} u_{\|}^{2} / 2}\left[e^{-x_{0}} \operatorname{Ei}\left(x_{0}\right)\right],
$$

where $X_{e}=\left(n_{e} / n_{T}\right) X$, with $n_{T}=n_{e}+n_{b}$. Also, $\alpha_{0}$ is the initial value of $\alpha$.

In the derivation of Eqs. (19) and (21) we have taken into account, the fact that the electrons are weakly relativistic, and have expanded the relativistic $\gamma$ factor as $\gamma \simeq 1+u^{2} / 2$. In the evaluation of the resonant part [Eq. (17)], where the exact position of the resonance curve is more important, relativistic effects have been fully incorporated.

\section{B. Evaluation of $/(n, m, I)$ and $e_{33}$ for the background distribution}

For the case of the background distribution, which remains stationary throughout the time evolution, the principal parts are given by similar expressions as those obtained for the energetic electrons

$$
\begin{aligned}
I^{\prime}(n, m, l)= & -\frac{4 \alpha_{b}^{2 m-2}}{\sqrt{\pi}} \int_{-\infty}^{\infty} d u_{\|} u_{\|}^{\prime} e^{-u_{\|}^{2} / \alpha_{b}^{2}}\left(\Theta(m) \sum_{k=0}^{m-1}(m\right. \\
& \left.-1-k) ! y_{n}^{k}-y_{n}^{m} e^{-y_{n}} E i\left(y_{n}\right)\right),
\end{aligned}
$$$$
e_{33}=\frac{4}{\alpha_{b}^{2}} \frac{X_{b}}{\sqrt{\pi}} \int_{-\infty}^{\infty} d u_{\|} u_{\|}^{2} e^{-\mu_{b} u_{\|}^{2} / 2}\left[e^{-y_{0}} E i\left(y_{0}\right)\right],
$$

where $\quad y_{n}=-\left[(2) /\left(\alpha_{b}^{2}\right)\right]\left(1-n Y-N_{\|} u_{\|}+u_{\|}^{2} / 2\right), \quad$ and $X_{b}=\left(n_{b} / n_{T}\right) X$.

The resonant parts for the case of background distribution can be obtained by the application of Eq. (17) to $f_{b}$ distribution. However, we further simplify the evaluation of these quantities by considering that for these very cold particles, $f_{b} \simeq A_{b} e^{-2 \gamma / \alpha_{b}^{2}}$, where $A_{b}=1 /\left[2 \alpha_{b}^{2} \pi K_{2}\left(2 / \alpha_{b}^{2}\right)\right], K_{2}$ being the modified Bessel function of the second kind. ${ }^{4}$ Therefore, the resonant part of $I(n, m, l)$ is simply given by

$$
\begin{aligned}
I^{\prime \prime}= & 2 \pi^{2} \mu_{b} A_{b} e^{-\mu_{b} n Y} \int_{u_{-}}^{u_{+}} d u_{\|} u_{\|}^{l} e^{-\mu_{b} N_{\|} u_{\|}} \mid\left[\left(n^{2} Y^{2}-1\right)\right. \\
& \left.+2 n Y N_{\|} u_{\|}-\left(1-N_{\|}^{2}\right) u_{\|}^{2}\right]^{m} .
\end{aligned}
$$

\section{NUMERICAL ANALYSIS AND RESULTS}

The relevant parameters for our quasilinear investigation are the ratio of electron plasma to electron cyclotron frequency, $\eta \equiv \omega_{p e} / \Omega_{e}$, the ratio between the energetic electron density and the background electron density, $\rho \equiv n_{e} / n_{b}$, the background electron temperature $T_{b}$, the effective temperature of the energetic electrons, $T_{e}$, the loss cone angle, and the ratio between wave energy and particle energy. We choose this set of parameters by considering a situation typical of the earth's auroral zone plasma.

The physical condition at the auroral region is such that hot magnetospheric population prevails over the cold one for $z \gtrsim 1.5 R_{E}$, where $R_{E}$ is the radius of the earth, while the cold population dominates at low altitudes. The effective temperature for the energetic electrons is never far from 4-5 $\mathrm{keV}$. It is known that the ratio of plasma to cyclotron frequency is lower than 0.1 for $1.1 R_{E} \leqslant z \leqslant 2 R_{E}$ and ap- 

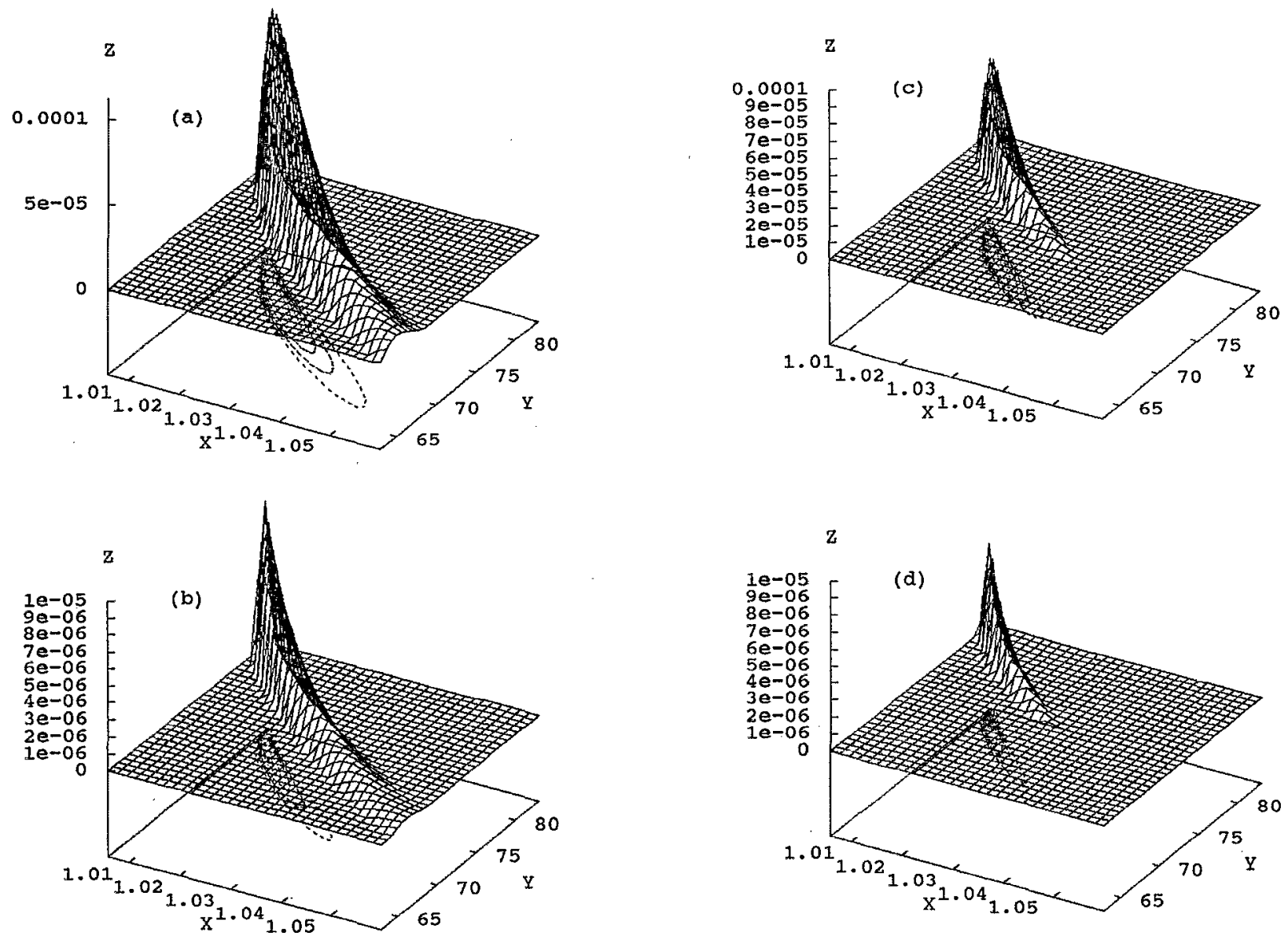

FIG. 2. (a) $X$ mode growth rate for $\Delta=0$ (initial value); (b) $O$ mode growth rate for $\Delta=0$ (initial value); (c) $X$ mode growth rate for $\Delta=0.2$; (d) $O$ mode growth rate for $\Delta=0.2$. $\omega_{p e} /\left|\Omega_{e}\right|=0.05, n_{b} / n_{e}=0.1 . x$ axis, normalized wave frequency $(w) ; y$ axis, $\cos ^{-1}(q)$.

proaches $\eta=1$ for $z \rightarrow 4 R_{E}$, and recent observations have emphasized the occurrence of low density plasmas $\left(n_{e}<1\right.$ $\mathrm{cm}^{-3}$ ) in the source region. ${ }^{45,46}$

Taking these informations into account, we assume a low density case, which may be relevant for the AKR (auroral kilometric radiation), by considering $\eta=0.05$. The background temperature is taken as $T_{b}=0.2 \mathrm{keV}$, the energetic temperature $T_{e}=4 \mathrm{keV}$, and the loss-cone angle is taken as $\theta_{\mathrm{LC}}=30^{\circ}$. We consider three different cases regarding the ratio of electron populations, ranging from the cold dominated case to the case of equal electron populations.

\section{A. Initial growth rates}

First we discuss the initial growth rates for each mode. For the present purpose, we concentrate on the fundamental $X$ and $O$ modes. The harmonics of these modes could be included in the analysis, in principle, but it turns out that they are only weakly unstable when compared with the fundamental modes, for the present choice of parameters. The $W$ mode is also stable. The $Z$ mode can play a very relevant role for higher density cases, ${ }^{40}$ but is stable for the present case.

Panels (a) and (b) of Fig. 2 show, respectively, the initial normalized growth rates for the $X$ and $O$ modes, for $\rho=0.1$, vs normalized frequency $w$ and $\cos ^{-1} q$. The quantity $\cos ^{-1} q$ approximately coincides with the propagation angle, for $w \simeq 1$ and refractive index $\simeq 1$, which is the case except near the cutoffs. We have searched the parameter space, and concentrated in the region where the growth rates are significant, utilizing a $31 \times 31$ grid in $w-q$ space. It is seen that absolute values of the growth rates are very small as compared to the real frequencies, which validates the use of quasilinear theory.

Panels (c) and (d) of Fig. 2 show the normalized growth rates for a case of partially filled loss cone, where we have assumed $\Delta=0.2$. These panels illustrate the decrease in the absolute value of the growth rates, and the reduction of the unstable region in $w-q$ space.

\section{B. Quasilinear analysis}

We now discuss the quasilinear evolution of the waves and particles. For this analysis, we start by discussing the initial wave spectral distribution. For the sake of simplicity, we assign the same initial level of wave energy for each mode. For the first set of applications, we assume that at $\tau=0, E_{\text {wave }}^{X}(0) / E_{\text {particle }}=E_{\text {wave }}^{O}(0) / E_{\text {particle }}=1.0 \times 10^{-4}$. In addition, we assume that the spectral electric field distribution is uniform over the initially unstable $(w, q)$ space, and zero, otherwise. The initial amplitude of the wave 


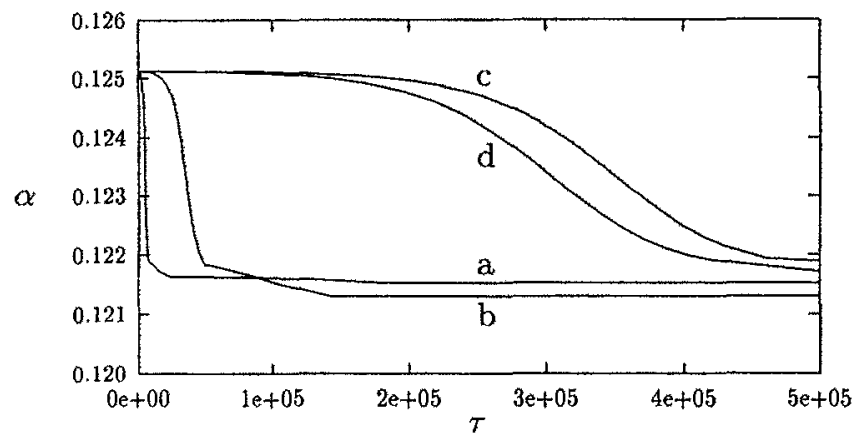

FIG. 3. Time evolution of the parameter $\alpha(\tau)$. (a) $\rho=1$; (b) $\rho=0.1$; (c) $\rho=0.01 . \omega_{\text {pe }} /\left|\Omega_{e}\right|=0.05, E_{\text {wave }} / E_{\text {particle }}(0)=1.0 \times 10^{-4}$, for each mode. Curve (d) was obtained with another approach, using cold plasma dispersion relation.

spectrum is then constant over unstable space, determined in such a way that the integrated spectrum satisfies the condition $E_{\text {wave }}^{X}(0) / E_{\text {particle }}=1.0 \times 10^{-4}$.

Using this procedure to determine the initial wave spectra, we have numerically solved the moment kinetic equations (6). These two equations, together with $\mathrm{Eq}$. (7) for the three modes of wave spectra, form a set of $3\left(n_{w} \times n_{q}\right)+2$ coupled equations, which we have solved by employing Runge-Kutta method with adaptative stepsize control, ${ }^{47}$ where $n_{w}$ and $n_{q}$ are the number of points in the spectral grid (we have used a $31 \times 31$ grid). In the solution of the dispersion relation we have neglected harmonics, and we have utilized the small Larmor radius approximation.

The resulting evolution of the parameters $\alpha(\tau)$ and $\Delta(\tau)$ vs the normalized time $\tau$ is depicted in Figs. 3 and 4 respectively, for three values of the population ratio, $p=0.01,0.1$, and 1.0. Figure 3 shows that the effective temperature $\alpha$ changes very little, attaining in the three cases an asymptotic value $\alpha(\tau \rightarrow \infty)=0.1215$. The difference between the three cases is the rate of change, with the steady state being quickly attained in the case of $\rho=1.0$, while the change is slowly produced in the case of $\rho=0.01$. In a different time

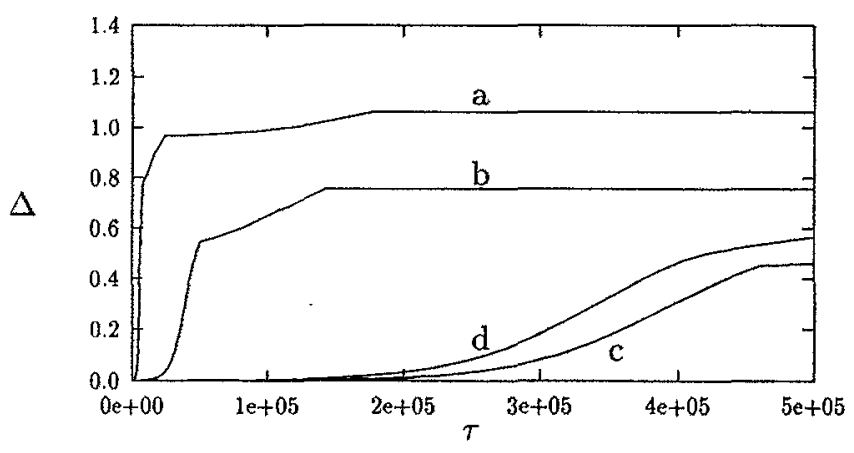

FIG. 4. Time evolution of the parameter $\Delta(\tau)$. (a) $\rho=1$; (b) $\rho=0.1$; (c) $\rho=0.01 . \omega_{p e} /\left|\Omega_{e}\right|=0.05, E_{\text {wave }} / E_{\text {particle }}(0)=1.0 \times 10^{-4}$, for each mode. Curve (d) was obtained with another approach, using cold plasma dispersion relation.

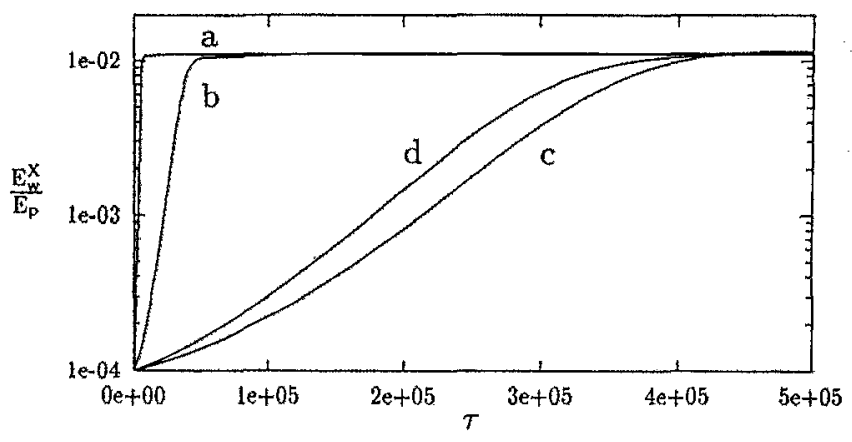

FIG. 5. Time evolution of $E_{\text {wave }}^{X} / E_{\text {particte }}$ (a) $\rho=\mathrm{I}$; (b) $\rho=0.1$; (c) $\rho=0.01$. $\omega_{p e} /\left|\Omega_{d}\right|=0.05, E_{\text {wave }} / E_{\text {particle }}(0)=1.0 \times 10^{-4}$, for each mode. Curve (d) was obtained with another approach, using cold plasma dispersion relation.

scale one sees that the case of $\rho=0.01$ is still evolving at time $\tau=5.0 \times 10^{5}$, with the asymptotic state of $\alpha \simeq 0.12128$ attained for $\tau=3.0 \times 10^{6}$. Curve (d) of Fig. 3 is for the case $\rho=0.01$, and is the outcome of a quasilinear analysis based upon an approximation which assumes that cold electrons support the waves. ${ }^{40}$

Figure 4 shows that in the case of $\rho=1.0$, the loss cone is quickly filled up, until the parameter $\Delta$ reaches $\Delta \approx 1.05$, for $\tau=1.8 \times 10^{5}$. The initial rate of evolution is smaller in the intermediate case, $\rho=0.1$, attaining the asymptotic state of $\Delta \simeq 0.75$. For the case of low population of energetic electrons, $\rho=0.01$, curve (c) of Fig. 4 shows that the asymptotic state of the quantity $\Delta$ is nearly $\Delta=0.5$, attained for $\tau$ even beyond $5 \times 10^{5}$. In Fig. 4(d) one can see the prediction based upon the cold plasma dispersion relation, featuring slightly larger loss-cone filling, as compared with Fig. 4(c).

In order to understand these results, one should note that the loss cone is already partially filled from the start by the cold electron population. In the case of smaller ratio between energetic and cold electrons a smaller amount of hot electrons diffusion into the loss cone is therefore sufficient to stabilize the instability, when compared to a case of larger population ratio. This explains why the saturation is attained for smaller values of $\Delta$ progressively when the population ratio decreases, as shown by curves (a), (b), and (c) of Fig. 4 .

Figure 5 displays the time evolution of $E_{\text {wave }} / E_{\text {particle }}$, for the $X$ mode. The case of $\rho=0.01$ is depicted by curve (c), which shows the wave amplitude initially growing slowly, and tending to saturate at a level of intensity approximately 2 orders of magnitude above the original wave level. The saturation indeed occurs beyond the time limit shown in Fig. 5, for $\tau \approx 3.0 \times 10^{6}$, with the asymptotic value of $E_{\text {wave }} / E_{\text {particle }} \simeq 1.286 \times 10^{-2}$. Figure 5(b) shows the case of $\rho=0.1$, which grows at a faster initial rate, but quickly saturates for $\tau=1.3 \times 10^{5}$. The case of equal electron populations is shown by Fig. 5(a). As a result of larger initial growth rates, the diffusion proceeds very quickly, but the instability saturates nearly at the same wave level attained in other cases of smaller population ratio, with asymptotic value slightly smaller than in the case of Fig. 5(b). Also shown is the result based upon the cold plasma dispersion relation, for $\rho=0.01$ [Fig. 5(d)]. The growth of the wave amplitude is 


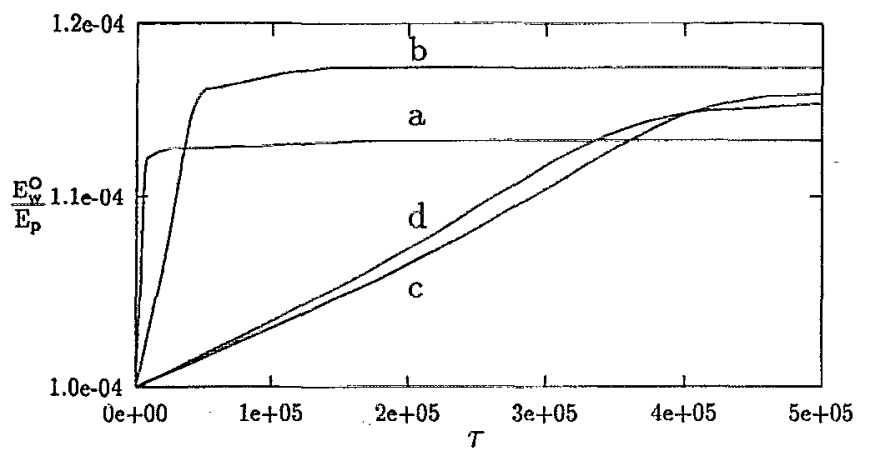

FIG. 6. Time evolution of $E_{\text {wave }}^{O} / E_{\text {particle }}$. (a) $\rho=1$; (b) $\rho=0.1$; (c) $\rho=0.01$. $\omega_{p e} /\left|\Omega_{c}\right|=0.05, E_{\text {tave }} / E_{\text {particle }}(0)=1.0 \times 10^{-4}$, for each mode. Curve (d) was obtained with another approach, using cold plasma dispersion relation.

initially slightly faster than predicted by the thermal code, but the asymptotic level is nearly the same.

The results depicted by curves (a), (b), and (c) in Fig. 5 deserve further comments. The case of smaller population of energetic electrons features smaller growth rates, which makes the wave growth slower. However, the saturation of the instability depends upon the diffusion, which fills up the loss cone. Due to the normalization utilized, according to Eq. (15), the amplitude of the wave energy in the case of $\rho=0.01$ is nearly a factor of 100 smaller than the amplitude in the case of $\rho=1.0$. The smaller amplitude of the wave energy explains the slower diffusion, which allows continued growth even after the instability has saturated for the case of higher energetic population.

Another interesting feature which must be discussed is connected with the conservation of energy in the amplification process. The conservation must be analyzed by comparing the average particle energy, as given by $\alpha$, and the wave energy, as given by the parameter $E_{\text {wave }} / E_{\text {particle }}$. The degree of filling up of the loss cone by energetic particles $(\Delta)$ is not directly involved in the energy conservation. It only contributes indirectly, through the average particle energy. It is seen from Fig. 5 that the asymptotic value of the energy ratio $E_{\text {wave }} / E_{\text {particle }}$ slightly increases with the decrease of the population ratio, while the quantity $\alpha$ decreases with the population ratio, as shown in Fig. 3. This behavior is as expected from wave-particle energy conservation.

The ordinary mode shows similar features as those obtained for the extraordinary mode. These features can be observed in Fig. 6, which shows the time evolution of $E_{\text {wave }} / E_{\text {particle }}$, for the $O$ mode. However, for the case of the ordinary mode, the growth is much less conspicuous, and the final wave level is between $10 \%$ and $15 \%$ above the original wave level. Similar to what we have in Fig. 5, curve (d) is obtained with the cold plasma code, for the same parameters as curve (c).

We also studied the influence of the initial level of wave activity, by considering different values of the ratio $E_{\text {wave }}(0) / E_{\text {particle }}$. Figure 7 shows the time evolution of the energy ratio for the $X$ mode, for $\rho=0.01$ and three different values of the initial ratio. In the case of very low initial activity $E_{\text {wave }}(0) / E_{\text {particle }}=1.0 \times 10^{-6}$, depicted by curve (c),

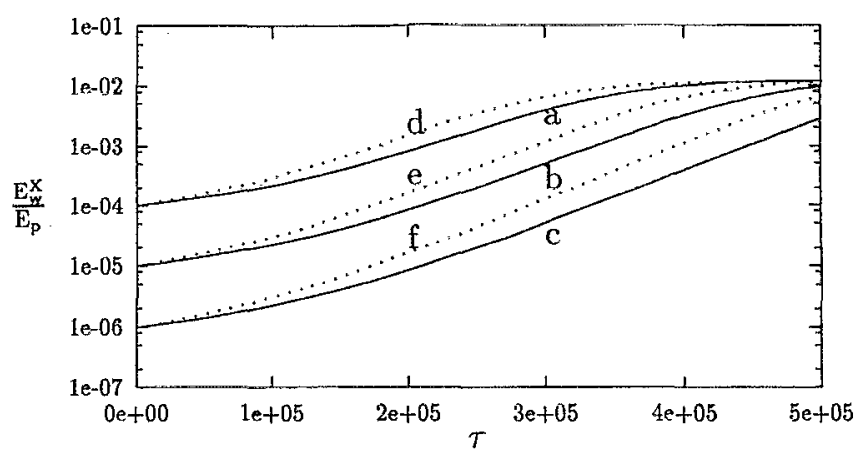

FIG. 7. Time evolution of $E_{\text {wave }}^{X} / E_{\text {particle }}$, for $\omega_{p e} /\left|\Omega_{e}\right|=0.05, \rho=0.01$, and three values of the ratio $E_{\text {wave }} / E_{\text {particte }}(0)$, for each mode. (a) $1.0 \times 10^{-4}$; (b) $1.0 \times 10^{-5}$; (c) $1.0 \times 10^{-6}$. Curves (d), (e), and (f) correspond to the same parameters as curves (a), (b), and (c), respectively, but were obtained with another approach, using cold plasma dispersion relation.

the diffusion proceeds slowly, while the wave level grows by a factor of 4 orders of magnitude. In the intermediate case, $E_{\text {wave }}(0) / E_{\text {particle }}=1.0 \times 10^{-5}$, shown by Fig. $7(\mathrm{~b})$, the growth is nearly of 3 orders of magnitude, while in the case of higher initial wave level [Fig. 7(a)], the growth is by a factor of 2 orders of magnitude.

Taking into account the results shown in Fig. 7, for different levels of initial wave activity, and also those of Fig. 5, one arrives at the conclusion that, for a significant range of initial wave levels and ratio of populations, the amplification due to the loss-cone instability, for $X$-mode waves, saturates at a level such that $E_{\text {wave }}(0) / E_{\text {particle }}(\tau \rightarrow \infty) \approx 1.0-2.0 \times 10^{-2}$. It is expected that this saturated level could be higher, however, if a source of loss-cone particles is taken into account in the kinetic equation. We have also included in Fig. 7 the results obtained with the formulation which uses the cold plasma dispersion relation. The qualitative results regarding the effect of the initial wave level are similar to those exhibited by Figs. 7(a) -7 (c). For the entire range of initial wave levels considered, the initial rate of wave growth predicted by the thermal calculation is smaller than that predicted by the cold plasma version, but the asymptotic level attained by the wave field is nearly the same in both cases.

Figure 8 shows the time evolution of the energy ratio for the $O$ mode, for $\rho=0.01$ and for the same three different values of the initial ratio as those considered in obtaining Fig. 7. The outcome is that the $O$ mode growth is not significant and remains on the order of $15 \%-25 \%$ growth over the initial value, for the whole range of initial wave level considered.

All these results were obtained for a fixed value of the parameter $\eta$, namely, $\eta=0.05$. However, it is interesting to verify the dependence on $\eta$ exhibited by our formulation, since previous studies based on an approximated analytical approach and numerical simulations have indicated that the efficiency of the amplification increases with the decrease of the frequency ratio $\eta .^{28}$ The saturation efficiency, denoted in Ref. 28 as $\epsilon$, was defined as the ratio between the average electromagnetic wave energy density at saturation and the perpendicular particle energy density. The distribution func- 


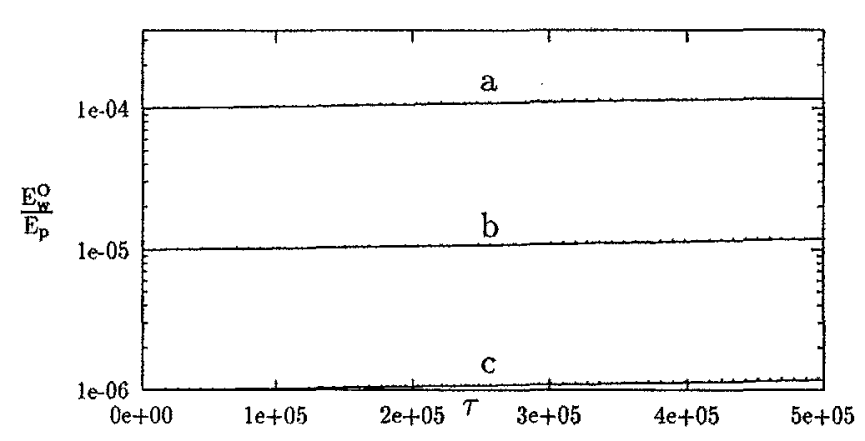

FIG. 8. Time evolution of $E_{\text {wate }}^{o} / E_{\text {particte }}$, for $\omega_{p e} /\left|\Omega_{e}\right|=0.05, p=0.01$, and three values of the ratio $E_{\text {wave }} / E_{\text {partacte }}(0)$, for each mode. (a) $1.0 \times 10^{-4} ;$ (b) $1.0 \times 10^{-5} ;$;c) $1.0 \times 10^{-6}$.

tion utilized as starting point for the simulations was a DGH distribution with loss-cone index $l$, assuming absence of the cold electron component.

Therefore, we have considered the case of $\eta=0.03$ and $\rho=1.0$, in order to compare our results with those of the numerical simulation of Ref. 28, regarding the $\eta$ dependence. In Table $I$ we list the saturation value of $E_{\text {wave }} / E_{\text {particle }}$, for the $X$ mode, for $\eta=0.03$ and $\eta=0.05$, and for $\rho=1.0$. We also list in Table I the saturation efficiency for the $X$ mode obtained in the numerical simulations described in Ref. 28 for the case $l=2$.

The ratio between the two values of $E_{\text {wave }} / E_{\text {particle }}$ listed in Table $I$ is $1.997 / 1.113 \simeq 1.79$. The ratio between the two values of the efficiency $\epsilon$ is $0.069 / 0.044 \approx 1.57$. On the other hand, the inverse ratio between the two values of $\eta$ considered is $0.05 / 0.03 \approx 1.67$. Therefore, we can conclude that the present quasilinear approach predicts a saturation efficiency for the $X$ mode which is nearly proportional to $\Omega_{e} / \omega_{\mathrm{pe}}$, similarly to what has been obtained with numerical simulations conducted in the same range of electron energies. ${ }^{28}$

\section{SUMMARY AND CONCLUSIONS}

In the present paper we have carried out a quasilinear analysis of the electron cyclotron maser instability driven by a loss-cone population. The novel feature of the present approach is that, instead of solving the quasilinear kinetic equation for the particles, we have modeled a time-dependent loss-cone distribution function, and discussed the evolution of the particles by only considering moments of quasilinear equation. This approach has already been used in another article based upon an approximation which assumes that the waves were supported by the cold plasma population, ${ }^{39,40}$ but the present analysis introduces thermal effects and utilizes the full dispersion relation in the quasilinear treatment of the

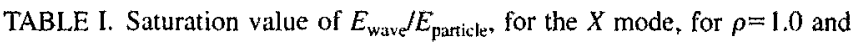
different values of $\eta$.

\begin{tabular}{ccc}
\hline \hline$\eta$ & $E_{\text {wave }} / E_{\text {particle }}$ & $\epsilon$ \\
\hline 0.05 & $1.113 \times 10^{-2}$ & 0.044 \\
0.03 & $1.997 \times 10^{-2}$ & 0.069 \\
\hline \hline
\end{tabular}

loss-cone instability. For the sake of demonstration, we have chosen a set of parameters typical of the earth's auroral zone plasma, which may be relevant to the description of the auroral kilometric radiation. For the parameters chosen, the ratio between electron plasma frequency and electron cyclotron frequency is sufficiently small in order that the only relevant unstable modes are the fundamental $X$ and $O$ modes.

We have initially considered the case of moderately high initial wave level, for three different ratios between the energetic and the background population. The results indicate that, even though the case of higher population of energetic electrons displays larger growth rates, the fast diffusion saturates the instability at a wave level which is not significantly different than that obtained in the case of small population of energetic particles. We have also studied the influence of the initial wave level, for a given ratio of populations. When the wave level is initially very small, the ensuing growth of $X$ mode waves is very significant, while it is only moderate in the case of relatively high initial wave level. For the $O$ mode, however, the wave growth has been demonstrated to be small for any initial wave level.

The dependence of the amplification efficiency on the frequency ratio $\omega_{\mathrm{pe}} / \Omega_{\mathrm{e}}$ has also been briefly discussed. It has been seen that the saturation amplitude for $X$ mode waves increases when this frequency ratio is decreased, in agreement with results previously obtained by means of numerical simulations.

We have also presented some results obtained with the use of an approximation which assumes that the cold electrons support the waves. In order to validate this approximation, we have considered the case of small population of energetic electrons, as compared to background electrons, for several values of the initial wave level. The resulting time evolution has been shown to be quantitatively similar to that obtained with the code which fully incorporates thermal effects, for the same population ratio.

The approach using the cold plasma approximation has been previously utilized for a case with higher value of the ratio between electron plasma to cyclotron frequency, such that the $Z$ mode was also initially unstable. For the case of dominant cold plasma, it has been seen that despite the fast initial growth for the $X$ mode, the $Z$ mode actually saturates at a larger amplitude. ${ }^{39.40}$ The method for calculating the growth rate introduced in the present paper is not particularly well suited for the $Z$ mode, because the $Z$ mode instability is an absolute instability with the group velocity approaching zero. However, if the limitation of the present method can be overcome (say, by directly calculating for the complex frequency), it would be interesting to investigate the situation in which the $Z$ mode competes with other modes, with the use of the code which fully includes thermal effects. The method presented in this paper can also be used for quasilinear analysis of the maser instability in the case of other applications, such as solar microwave bursts.

\section{ACKNOWLEDGMENTS}

Numerical computations were performed at the Supercomputer Center of the Universidade Federal do Rio Grande 
do Sul (CESUP-UFRGS). One of the authors (L. F. Z.) acknowledges fruitful discussions with R. Gaelzer. Another author (P. H. Y.) acknowledges the hospitality of the Institute of Physics at UFRGS and CESUP-UFRGS during his visit to Porto Alegre, Brazil.

This work has been partially supported by the Brazilian agencies Conselho Nacional de Desenvolvimento Científico e Tecnológico ( $\mathrm{CNPq})$ and Financiadora de Estudos e Projetos (FINEP).

'D. E. Gary, J. L. Linsky, and G. A. Dulk, Astrophys. J. L 79, 263. (1982).

${ }^{2}$ K. A. Lang and R. F. Wilson, Astrophys. J. 305, 6423 (1986).

${ }^{3}$ P. H. Yoon and T. Chang, Astrophys. J. 343, 31 (1989).

${ }^{4}$ R. G. Hewitt, D. B. Melrose, and K. G. Rönmark, Proc. Astron. Soc. Aust. 4, 226 (1981).

${ }^{5}$ H. P. Ladreiter and Y. Leblanc, J. Geophys. Res. 95, 367 (1990).

'P. Galopeau, P. Zarka, and D. Le Quéau, J. Geophys. Res. 94, 8739 (1989).

${ }^{7}$ S. A. Curtis, Nature (London) 318, 47 (1985).

${ }^{8}$ Y. Leblanc, Adv. Space Res. 10, 39 (1990).

${ }^{9}$ G. D. Holman, D. Eichler, and M. R. Kundu, in Proceedings of the IAU Symposium on Radio Physics of the Sun, edited by M. R. Kundu and T. E. Gergely (Reidel, Dordrecht, 1980), Vol. 86, p. 457.

${ }^{10}$ D. B. Melrose and G. Dulk, Ástrophys. J. 259, 844 (1982).

${ }^{11}$ M. J. Aschwanden and A. O. Benz, Astrophys. J. 332, 447 (1988).

${ }^{12}$ M. J. Aschwanden, Astron. Astrophys. 237, 512 (1990).

${ }^{13}$ M. Güdel, Astron. Astrophys. Lett. 1, 239 (1990).

${ }^{14}$ C. S. Wu and L. C. Lee, Astrophys. J. 230, 621 (1979).

${ }^{15}$ C. S. Wu, S. T. Tsai, M. J. Xu, and J. W. Shen, Astrophys. J. 248, 384 (1981).

${ }^{16}$ N. Omidi and D: A. Gurnett, J. Geophys. Res. 89, 10801 (1984).

${ }^{17}$ H. K. Wong, C. S. Wu, F. J. Ke, R. S. Schneider, and L. F. Ziebell, J. Plasma Phys, 28, 503 ,(1982).

${ }^{18} \mathrm{C}$. S. Wu, H. K. Wong, D. J. Gorney, and L. C. Lee, J. Geophys. Res. 87, 4476 (1982). $A$

${ }^{19}$ D. B. Melrose, K. G. Rönmark, and R. G. Hewitt, J. Geophys. Res. 87, 5140 (1982).

${ }^{20} \mathrm{P}$. B. Dusenbury and L. R. Lyons, J. Geophys. Res. 88, 10072 (1983).

${ }^{21}$ C. S. Wu and X. M. Qiu, J. Geophys. Res. 89, 883 (1984).
${ }^{22}$ N: Omidi, C. S. Wu, and D. A. Gurnett, J. Geophys. Res. 89, 883 (1984).

${ }^{23}$ D. B. Melrose, R. G. Hewitt, and G. A. Dulk, J. Geophys. Res. 89, 2831 (1984).

${ }^{24}$ D. Le Quéau, R. Pellat, and A. Roux, J. Geophys. Res. 89, 897 (1984).

${ }^{25}$ D. Le Quéau, R. Pellat, and A. Roux, Phys. Fluids 27, 247 (1984).

${ }^{26}$ D. Le Quéau and P. Louarn, J. Geophys. Res. 90, 2605 (1989).

${ }^{27}$ N. Omidi and C. S. Wu, J. Geophys. Res. 90, 6641 (1985)...

${ }^{28}$ P. L." Pritchett, Phys. Fluids 29, 2919 (1986).

${ }^{29}$ C. S. Wu, Space Sci. Revi. 41, 215 (1985).

${ }^{30}$ J. S. Wagner, L. C. Lee, C. S. Wu, and T. Tajima, Geophys. Res. Lett. 10, 483 (1983).

${ }^{31}$ J. S. Wagner, L. C. Lee, C. S. Wu, and T. Tajima, Radio Sci. 19, 509 (1984).

*.32 P. L. Pritchett and R. J. Strangeway, J. Geophys. Res. 90, 9650 (1985).

${ }^{33}$ P. L. Pritchett and R. M. Winglee, J. Geophys. Res. 94, 129 (1989).

${ }^{34}$ R. Gaelzer, L. F. Ziebell, and R. S. Schneider, J. Geophys. Res. 97, 19299 (1992)...;

${ }^{35}$ R. Gaelzer, L. F. Ziebell, and R. S. Schneider, J. Geophys. Res. 99, 8905 (1994).

${ }^{36}$ P. H. Yoon and C. S. Wu, Phys. Rev. A 4, 6819 (1991).

${ }^{37}$ H. S. Uhm, R. C. Davidson, and K. R. Chu, Phys. Fluids 21, 1866 (1978).

${ }^{38}$ H. S. Uhm and R. C. Davidson, Phys. Fluids 29, 2713 (1986).

${ }^{39}$ L. F. Ziebell and P. H. Yoon, in Segundo Encontro Brasileiro de Física de Plasmas (Brazilian Physics Society, Serra Negra, Brazil, 1993), p. 365.

${ }^{40}$ P. H. Yoon and L. F. Ziebell, "Quasilinear evolution of cyclotron maser instability," to he published in Phys. Rev. E.

${ }^{41}$ L. F. Ziebell, J. Plasma Phys. 39, 431 (1988).

${ }^{42}$ L. F. Ziebell and G. Granata, Phys. Fluids 29, 3730 (1986).

${ }^{43}$ Handbook of Mathematical Functions, edited by M. Abramowitz and I. A. Stegun (Dover, New York, 1970), p. 228.

${ }^{44}$ Handbook of Mathematical Functions, edited by M. Abramowitz and I. A. Stegun (Dover, New York, 1970), p. 374.

${ }^{45}$ P. Louarn, A., Roux, H. de Féraudy; D. Le Quéau, M. André, and L. Matson, J. Geeophys. Res. 95, 5983 (1990):

${ }^{46}$ A. Hilgers, B. Holback, G. Homgren, and R. Boström, J. Geophys. Res. 97, 8631 (1992).

${ }^{47}$ W. H. Press, B. P. Flannery, S. A. Teukolsky, and W. T. Vetterling, Numerical Recipes: The Art of Scientific Computing (Cambridge University Press, Cambridge, 1989), p. 554. 